\title{
La indeterminación de la traducción archivística
}

\author{
Por Alejandro Delgado Gómez
}

Resumen: La archivística está sufriendo un cambio radical, frecuentemente escrito en inglés. Para comunidades no anglo-parlantes implica la necesidad de una traducción de los textos archivísticos más relevantes. Sin embargo, esto plantea varios retos y dificultades: diferentes comunidades disciplinares, diferentes usos de los términos por parte de diferentes comunidades anglo-parlantes, y diferentes comprensiones, asociadas a diferentes tradiciones nacionales. A este respecto, este texto sugiere varios ejemplos y riesgos potenciales derivados de una comprensión mala o pobre. Del mismo modo, explora algunas de las respuestas propuestas por investigadores archivísticos, especialmente los ensayos publicados por Eric Ketelaar. De acuerdo con estos fundamentos, y sobre la base de la teoría de la indeterminación de Quine, este texto sugiere dos aproximaciones al problema: un sólido estudio etnográfico de los entornos socio-culturales asociados a diferentes términos y discursos archivísticos, y un profundo análisis conceptual de algunos de los conceptos más relevantes de la archivística moderna.

Palabras clave: Análisis conceptual, Contexto, Discurso archivístico, Etnografía archivística, Indeterminación de la traducción, Traducción.

\section{Title: The indeterminateness of archival translation}

Abstract: Archival science is suffering a radical change, often written in English. For non English-speaking communities, this implies the need for a translation of the most relevant archival texts. However, this poses several challenges and difficulties: different disciplinary communities, different uses of terms by different English-speaking communities, and different understandings, associated to different national traditions. In this regard, this text sug-

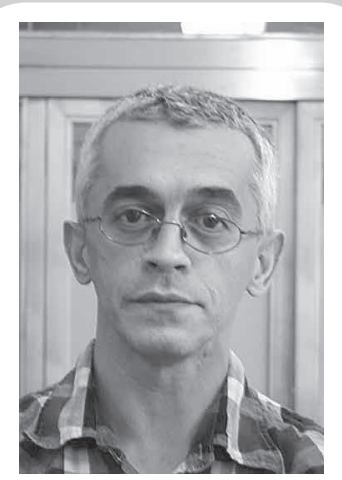

Alejandro Delgado Gómez es técnico de archivo y biblioteca del Ayuntamiento de Cartagena. Consultor de 3000 Informática. Investigador afiliado del Center for Information as Evidence de UCLA. Ha sido observador invitado del Proyecto InterPares 2, para el que traduce sus documentos, así como habitualmente del Records Continuum Research Group gests several examples, as well as potential risks derived from a bad or poor understanding. In the same way, it explores some of the answers proposed by archival researchers, particularly essays published by Eric Ketelaar. According to this background, and on the basis of the Quine's theory of indeterminateness, this text suggests two approaches to the problem: a sound ethnographical study of socio-cultural environments associated to different archival terms and discourses, as well as a deep conceptual analysis of some of the most relevant concepts in modern archival science.

Keywords: Archival discourse, Archival ethnography, Conceptual analysis, Context, Indeterminateness of translation, Translation.

Delgado Gómez, Alejandro. "La indeterminación de la traducción archivística”. En: El profesional de la información, 2007, enero-febrero, v.16, n. 1, pp. 39-46.

\section{Introducción}

De unos años a esta parte, la disciplina y la práctica archivísticas han venido experimentando un vuelco radical que se ha escrito, hasta donde sabemos, en gran medida, pero no exclusivamente, en lengua inglesa, tanto porque las comunidades originales son angloparlantes como, en menor grado, porque los colectivos en otras lenguas han venido a considerarla como lingua franca. Esto no significa, por otra parte, que todos los grupos archivísticos anglo-parlantes hayan adoptado el mismo cambio de modelo ni en el mismo grado. En dicho conjunto de comunidades existe en la actualidad un amplio abanico conceptual y práctico que abarca desde un modelo estrictamente tradicional del ciclo de vida de los documentos hasta las aproximaciones más evolucionadas al modelo del continuo de los documentos.

En un marco social y profesional crecientemente globalizado, se puede concebir de manera argumentable la necesidad de transmitir este vuelco radical a otras comunidades que se desenvuelven en otras lenguas. En lo que nos concierne, es posible pensar en la necesidad de transmitirlo a la comunidad archivística que trabaja en lengua castellana. De hecho, distintos grupos e individuos están haciendo en la actualidad el esfuerzo de traducir al castellano normas y textos relevantes a partir de los originales en lengua inglesa. 
Sin embargo, esto ha planteado debates y discusiones acerca de la oportunidad de la utilización de ciertos términos en lugar de otros, más familiares a la tradición de destino. El problema, a nuestro juicio, tiene múltiples facetas. Por una parte, el modelo archivístico que se está articulando en los originales en lengua inglesa trata de reflejar una realidad documental e informativa compleja, interdisciplinar y en muchas ocasiones nueva, para cuya expresión se requiere con frecuencia el uso de términos y conceptos también complejos, interdisciplinares y nuevos, incluso en el original. Por otra, las muy diversas tradiciones en lengua inglesa están ellas mismas enzarzadas en un debate acerca del modo en que debieran utilizarse los términos, el significado de cada uno de ellos y los conceptos que reflejan. Además, esta discusión entre tradiciones en una sola lengua se multiplica y crece cuando se asume el esfuerzo de transmitir los hallazgos realizados en un idioma y dentro de una tradición a otra lengua y dentro de otra tradición posiblemente muy diferente y desarrollada en un marco socio-cultural distinto. Por último, el debate acerca del modo de traducir se está enfocando sobre los términos, más que sobre el discurso en el que éstos aparecen. Si bien una correspondencia término a término es útil, rara vez tienen sentido aislados; más bien lo adquieren en un determinado contexto, en combinación con otros términos y como medio de expresión de ideas.

Por supuesto, es muy probable que este problema sea relevante, no sólo a la disciplina y la práctica archivísticas, sino a todas las disciplinas del ámbito de la información y, más allá de las ciencias sociales, de las ciencias naturales, de la literatura y las artes. Este supuesto, aunque plausible, excede el alcance de nuestra competencia.

El presente texto aborda los problemas asociados a la traducción de textos archivísticos escritos en una lengua y en el marco de una tradición a otra lengua y en el contexto de otra tradición quizá muy diferente. Además, aborda los problemas asociados a la traducción de términos desarrollados en el marco de una tradición a otra que opera con la misma lengua; así como los problemas asociados a la traducción de términos desarrollados en el marco de una disciplina dada a otra que opera en el marco de la misma tradición y la misma lengua. Para ello, el presente texto asume la teoría de la indeterminación radical de la traducción, propuesta por el filósofo estadounidense Willard van Orman Quine, así como la hipótesis del teórico de la archivística Eric Ketelaar, relativa a la necesidad de comprender los contextos socio-culturales más amplios en los que se desenvuelve una tradición archivística dada, antes de proceder a equiparar los términos en los que se expresa esta tradición y, por debajo de tales términos, los significados y conceptos que comportan.
El presente texto expone en primer lugar algunos ejemplos que proceden de lo general a lo específico:

- Acerca del problema asociado a la utilización de un término en diferentes contextos disciplinares en una sola lengua.

- Sobre el problema asociado a la utilización de un término en el contexto disciplinar de la archivística en una sola lengua pero en diversas tradiciones.

- En relación con el problema asociado a la traducción de un término utilizado en el contexto disciplinar de la archivística en una lengua y una tradición dadas a otra lengua y otra tradición.

En segundo lugar, expone someramente algunas de las soluciones que se han propuesto a tales problemas. Por último, esboza su propia propuesta de solución, si es que tal solución existe.

\section{Un discurso interdisciplinar}

Las disciplinas de la información, y entre ellas la archivística, se enfrentan en la actualidad, y pese a la especificidad de cada una de ellas, a una amplia gama de problemas comunes, derivados en gran parte del uso creciente de nuevas tecnologías que vienen recibiendo las denominaciones de tecnologías de la información y de convergencia universal ${ }^{1}$. Por ejemplo, aunque la especificidad de la archivística reside básicamente en el hecho de que su objeto de interés es la información registrada en forma de documento, o de objeto asimilable a documento, así como la propiedad de evidencia a efectos de responsabilidad y memoria de estos documentos u objetos asimilables, los profesionales de la archivística se enfrentan a retos similares a aquellos que tienen los de la biblioteconomía o la museología a la hora de, digamos, pluralizar sus respectivos objetos sobre la Red, o para proporcionar continuidad a lo largo del tiempo a sus objetos digitales. Prueba de ello sería el creciente interés en, y el consiguiente desarrollo de, los estudios interdisciplinares ${ }^{2}$. Estos estudios, a mayor abundancia, no se enfocan exclusivamente sobre las tradicionales profesiones de la información, sino que de manera creciente implican a disciplinas muy diversas - desde la sociología y la filosofía hasta la gestión de empresas, pasando por el derecho, la lingüística, la antropología, la etnografía, las matemáticas, e incluso la literatura de ciencia ficción- con la esperanza de encontrar respuestas a los múltiples problemas derivados del entorno digital a partir del uso imaginativo de otros conceptos, procedimientos y técnicas.

Esta perspectiva interdisciplinar es sin duda extremadamente enriquecedora y, como se espera mostrar en una sección posterior, aporta realmente soluciones; sin embargo, debe preverse la posibilidad de que el uso 
de un término, y del concepto subyacente, en un determinado marco disciplinar sea diferente del uso del mismo término en otro contexto. Como se ha dicho, cada disciplina tiene su propia especificidad y ha evolucionado de distinta manera, y el hecho de que diferentes áreas utilicen términos idénticos no implica que tengan siempre el mismo significado. Un ejemplo convencional es "clasificación", cuyo uso es distinto, digamos, en el ámbito de las bibliotecas y en el de los archivos.

\section{"El hecho de que diferentes áreas utilicen términos idénticos no implica que tengan siempre el mismo significado"}

Términos especialmente complejos son el de "documento" y, por lo que se refiere a los intereses archivísticos, el de "evidencia" y sus asociados "responsabilidad" y "memoria". "Documento", en el contexto de una biblioteca, carece del matiz jurídico que tradicionalmente se le atribuye en el contexto de los archivos. Para una biblioteca, los documentos no comportan "evidencia" a efectos de "responsabilidad", aunque algunos de ellos (por ejemplo, un diario o la publicación de la correspondencia de un autor), pueden comportar "evidencia" a efectos de "memoria". No obstante, cabe argumentar de manera concebible que esta "evidencia" a efectos de "memoria" no es el objeto de interés de la biblioteca, sino más bien del editor o de la crítica literaria.

En este sentido, "documento", desde la perspectiva del archivo, estaría más próximo al punto de vista del derecho que el de la biblioteca. Sin embargo, dentro de la especialidad de la archivística etnográfica o de la archivística oral, "documento" carecería en principio del matiz jurídico y estaría más próximo al término "documento" tal y como se usa en antropología, aunque ciertamente sería "evidencia" a efectos de "memoria". Adicionalmente, si este documento se utilizara (como de hecho ha sucedido en países de triste pasado, como Sudáfrica, Australia o los países de la órbita soviética) en un tribunal como testimonio de derechos, la "evidencia" del "documento" antropológico pasaría a adquirir un nuevo significado, ya no sólo como portador de "memoria", sino también como portador de "responsabilidad".

De manera breve, el término "documento", como el objeto al que representa, no tiene un solo significado; más bien, adquiere uno nuevo cada vez que se le instancia en un determinado contexto de uso: una biblioteca, un tribunal, un periódico, un archivo de colecciones, un archivo activo, etc.

La discusión precedente no es mero entretenimiento bizantino. En el discurso interdisciplinar actual se tiende a interpretar que un documento, en el sentido en el que se utiliza el término en archivos, puede tratarse como un documento en términos generales, o como información, o como datos. Si bien los documentos contienen datos y forman parte de sistemas de información o de conocimiento más amplios, una insuficiente dilucidación del término, y del concepto asociado, "documento" puede conducir a la eliminación de la propiedad fundamental de evidencia en el documento, y de sus valores como responsabilidad y memoria ${ }^{3}$.

\section{Diferentes tradiciones}

En el marco de una sola lengua, de manera especial, evidentemente si es de uso en más de un ámbito nacional, un solo término puede tener significados diferentes, dependiendo de las diversas tradiciones nacionales. Brevemente, y por utilizar un ejemplo muy significativo, la archivística en lengua inglesa ha evolucionado en dos direcciones diferentes que sólo en los últimos años han llegado a confrontarse. Básicamente, la tradición archivística anglófona deriva de la traducción del manual de los archiveros holandeses Muller, Feith y Fruin $^{4}$, que todavía se sigue citando en el ámbito educativo. A partir de este manual, el archivero británico Sir Hilary Jenkinson ${ }^{5}$, poco conocido y utilizado en nuestro país, desarrolló su concepto de "santidad de la evidencia", con un matiz abiertamente conservacionista y no intervencionista. Posteriormente, el archivero norteamericano Theodore R. Schellenberg propuso la conocida y algo arbitraria discriminación de los documentos dependiendo del momento en que se encontraran dentro de su ciclo de vida ${ }^{6}$. Esto implicó la creación de dos disciplinas: archives y records management, con un matiz ligeramente intervencionista la segunda de ellas, y declaradamente conservacionista la primera. Esta teoría, filtrada de diversas maneras, es la que ha llegado a ser mejor conocida en Europa. Sin embargo, en la misma década en la que Schellenberg formulara su teoría, algunos archiveros australianos, y significativamente Ian Mclean ${ }^{7}$, retomaban el concepto jenkinsoniano de evidencia y comenzaban a elaborar un modelo teórico muy diferente que a principios de los años noventa del siglo veinte sería articulado por investigadores archivísticos como Upward y McKemmish en lo que se ha venido a conocer como el modelo del continuo de documentos ${ }^{8}$ que, entre otras muchas complejidades, no discrimina archives y records management. Antes al contrario, hace uso del término global recordkeeping, significando con el mismo la disciplina de la gestión de documentos, tanto si están destinados a durar un nanosegundo como si están diseñados a durar milenios ${ }^{9}$.

La crisis del modelo del ciclo de vida, no sólo en entornos técnicos cada vez más digitales, sino también 
en contextos políticos y sociales crecientemente irresponsables; así como la fascinante globalidad del modelo del continuo de documentos, han traído este último a primer plano como potencial sustituto del modelo del ciclo de vida. Entre los muchos debates que este cambio de paradigma ha suscitado, aparece un modesto debate terminológico, a saber, la oportunidad de la utilización del término recordkeeping en lugar de records management. Desde el punto de vista de la tradición estadounidense, esta confrontación es exacta, por cuanto en los EUA ambos términos se utilizan en el contexto de los documentos llamados vivos. Sin embargo, desde el punto de vista de la tradición australiana, el debate debiera plantearse en términos de recordkeeping, por una parte y archives/records management por la otra ${ }^{10}$. Si esto es así, la acidez del debate está más que justificada, puesto que se deja de hablar de un simple cambio terminológico y la discusión se convierte en discusión acerca de un cambio global de modelo discursivo y, más allá, de un cambio global de paradigma archivístico que, en principio, afecta a cuestiones tan esenciales para una disciplina como la modificación de la manera en que se ejecutan algunas de sus técnicas cotidianas (la descripción, la clasificación, la evaluación o la distribución) la redefinición de algunos de sus principios básicos (el principio de procedencia y el de orden original) o la re-elaboración de sus conceptos esenciales (el de documento). Desde la perspectiva de la tradición estadounidense del ciclo de vida, un documento es un objeto pasivo sobre el que se ejecutan tareas a medida que va cumpliendo etapas. Desde la perspectiva australiana es un agente activo que está siempre en estado de llegar a ser, y que participa en un complejo continuo de relaciones con otros agentes y funciones.

Es decir, en la actualidad los archiveros estadounidenses y los australianos están utilizando los mismos términos (archives, recordkeeping, records management, record o document), pero no están hablando en absoluto de lo mismo. No solamente no están hablando de los mismos conceptos, sino que ni siquiera están hablando de una sola manera de concebir la archivística.

\section{Problemas con la traducción}

El ejemplo anterior debiera sugerir alguno de los problemas a los que se enfrenta el traductor de un texto archivístico que intenta transmitirlo con cierta exactitud en una lengua diferente a la original. Tradicionalmente, records management se ha traducido al castellano como "gestión de documentos", lo cual es bastante exacto, a pesar de que en la tradición archivística española no existe la discriminación archivero/gestor de documentos. Sin embargo, recordkeeping, ya sea en la vertiente americana como en australiana, también ha comenzado a traducirse como "gestión de documentos", dando lugar a un potencial malentendido: el de que el objeto y los procedimientos de la gestión de documentos, recordkeeping, son equivalentes al objeto y a los procedimientos de la gestión de documentos, records management, lo cual es bastante inexacto. Recordkeeping, en acepción australiana, es una disciplina global que estaría mucho más cerca de la concepción española de la disciplina archivística abarcando todas las edades de los documentos, que de la concepción norteamericana del records management, por una parte, dedicado a los documentos de uso activo; y archives, por otra, dedicados a los documentos de valor llamado permanente.

Sin embargo, una traducción simple de recordkeeping como "archivística" tampoco sería correcta. En el contexto australiano del continuo de los documentos, abarca la dimensión de la creación de éstos, que está ausente de la tradición archivística española, de tal manera que una traducción simple daría lugar a otro potencial malentendido, el de que la tradición australiana también excluye de su objeto de trabajo la dimensión de creación, con consecuencias para la comprensión del modelo de evidencia, responsabilidad y memoria que se maneja, y que está adquiriendo cada vez mayor relevancia a nivel internacional. Por lo demás, ni siquiera en la tradición de origen los términos están aún bien aquilatados, de manera que es posible encontrar en un solo texto la ocurrencia de recordkeeping, archives $\mathrm{y}$, con menor frecuencia, records management.

\section{"Cuando en Suecia se habla de un sistema de registro no se está diciendo lo mismo que cuando se hace en el Reino Unido, ni tampoco es comparable al caso de España"}

Existen otros ejemplos convencionales procedentes de diversos contextos. Digamos que cuando en Suecia se habla de un sistema de registro no se está diciendo lo mismo que cuando se hace en el Reino Unido, ni tampoco es comparable al caso de España. El uso del término "sistema de registro" se ha venido consolidando, dentro de diferentes tradiciones, a partir de la diversa recepción, o ausencia de la misma, del registraturprinzip de origen prusiano; de la combinación de este principio con otros históricamente fundamentados, como el de procedencia, el de orden original o el antiguo principio francés de pertinencia ${ }^{11}$; y de la combinación de todo ello con las peculiaridades socio-culturales de cada nación. Tal y como ha explicado Eric Ketelaar, no existe nada semejante a una concepción europea de la archivística $^{12}$. Una prueba de ello debiera ser el esfuerzo por mantener la neutralidad por parte de especificaciones 
como MoReq que, en su actual propuesta de revisión, hace patente tanto la necesidad de devenir compatible con una norma que se esfuerza en ser neutral (la ISO 15489-Gestión de documentos ${ }^{13}$ ), como la necesidad de revisar y si es preciso incorporar los hallazgos de diferentes tradiciones nacionales ${ }^{14}$.

Con todo lo dicho, aún existe un par de términos que tradicionalmente ha planteado serios problemas al traductor del inglés al castellano, y al que se debiera hacer mención, siquiera por encima. Se trata del par document/record. En el original, el primer término se ha definido convencionalmente como "información registrada", mientras que el segundo se ha entendido convencionalmente como "información registrada que además constituye evidencia a efectos de responsabilidad"15. En castellano no existe esta discriminación, digamos, interna al término. En nuestra tradición se ha utilizado un solo término, "documento", para expresar ambos significados, y la discriminación ha venido dada por el contexto de uso (archivístico, bibliotecario, jurídico, etc.). Además, y aunque el intento de hacer valer derechos históricos en contextos contemporáneos no resulta un expediente muy adecuado, parece ser que el matiz jurídico del término "documento" es anterior al matiz bibliográfico y simultáneo al educativo, y que, de hecho, hasta bien avanzado el siglo XIX, las compilaciones de documentos no jurídicos recibían el nombre de "monumenta", como medio de discriminación ${ }^{16}$.

Convencionalmente, en el momento de traducir $d o$ cument y record se utilizaba sólo el término "documento" $\mathrm{o}$, si se consideraba necesaria una indicación adicional, se traducía document como "documento" y record como "documento de archivo". La aparición del cuerpo normativo ISO 9000 implicó la traducción de record como "registro", medio de discriminación que ya se había utilizado puntualmente con anterioridad en castellano ${ }^{17}$. En principio, este refinamiento adicional sobre la traducción debiera resultar útil a efectos de especificidad; sin embargo, en el contexto de la archivística plantea un nuevo problema, el de la homonimia "registro" (documento) y "registro" (sistema de registro). A pesar de lo cual, se podría argumentar de manera concebible que la traducción como "registro" está etimológicamente más próxima a record de lo que lo está la traducción como "documento" o "documento de archivo". "Registro" procede del singular regestum, que justificaría el uso del término en su acepción "sistema de registro"; pero, más allá, regestum deriva a su vez de regerere, con varios significados: por supuesto, el de recolectar, apilar o amontonar, tal y como harían un sistema de registro o un archivo simples; pero también el de recordar y el de soportar un escrutinio, es decir, los dos valores de la evidencia del documento. Además, y muy significativamente, record procede del latín recordari, es de- cir, "recordar". Document y "documento" proceden del latín documentum, con el significado más restrictivo de "documento oficial” y "prueba"; más allá, documentum procede a su vez de docere, adquiriendo por tanto también el significado de "ejemplo para la enseñanza", que se aleja de los intereses de la archivística ${ }^{18}$.

Básicamente, lo que se trata de mostrar es que, de la combinación de la perspectiva histórica y etimológica, la traducción de record como "documento", "documento de archivo" o "registro" es indecidible. Como se intentará explicar en la sección posterior, las tres traducciones son igualmente legítimas, dependiendo del contexto del discurso, y la elección de uno u otro término dependerá de cuestiones tales como la distancia de aparición de los términos originales record, document o registry; de la opinión del autor del original al respecto; del potencial uso variable de los términos dentro del original; e incluso de factores estéticos y subjetivos, como la calidad literaria del original o la ocurrencia de cacofonías.

\section{Algunas propuestas de solución}

Anne Gilliland, a propósito del actual debate acerca de si metadatos y descripción archivística son dos maneras de expresar la misma técnica, ha analizado el problema de la reconciliación del uso de diferentes términos en diversas tradiciones y en una sola lengua para referirse a conceptos similares. De acuerdo con la autora, y en el marco del proyecto InterPares 2, es necesaria la compilación de un vocabulario que refleje sin ambigüedad conceptos y procesos similares. No obstante, debiera estar dotado de un grado suficiente de granularidad, como para permitir que queden registrados en él todos los posibles matices de términos complejos, como el de metadatos ${ }^{19}$.

En una línea semejante, es decir, el establecimiento de concordancias entre términos utilizados por diferentes tradiciones para reflejar conceptos, si no idénticos, al menos similares, Jean Dryden lleva a cabo el escrutinio de los esfuerzos realizados en la última mitad del siglo veinte y comienzos del veintiuno para normalizar, por una parte, la terminología archivística en lengua inglesa y, por otra, las concordancias terminológicas entre diversos idiomas. A partir de un resultado poco optimista, y con la finalidad declarada de mostrar que la archivística sólo podrá reclamar su derecho a llamarse profesión cuando pueda articular un cuerpo de conocimiento profesional expresado en un discurso normalizado, Dryden propone el uso de las técnicas avanzadas de la disciplina terminológica para llegar a un acuerdo acerca del vocabulario a utilizar por la profesión ${ }^{20}$.

Sin embargo, las aproximaciones al problema basadas exclusivamente en la terminología, con un matiz 
teórico positivista, tienen la doble limitación de que, por una parte, conciben la realización de un emparejamiento perfecto entre términos, algo que ya se ha visto que no siempre es posible; y, por otra que olvidan que, cuando se traduce, rara vez se hace un término aislado, sino un discurso completo que plantea el reto de decidir entre varias opciones posibles atendiendo a criterios como los mencionados más arriba. Es decir, y teniendo en cuenta las diferencias culturales de distintas tradiciones, si la traducción se planteara simplemente en cuestión de términos, es probable que no pudiera traducirse nada, ya no sólo en el ámbito de la archivística, sino en cualquier otro. Por ejemplo, algunas culturas esquimales utilizan hasta nueve términos para nuestro término "nieve"; sin embargo, en swahili no existe ningún término para esta realidad; lo que de acuerdo con los zulúes se conoce como color rojo no es lo mismo que codifica un hispano-hablante como rojo; los indios navajos identifican conceptual, y por tanto también gramaticalmente, agente y acción; y para la cultura hopi la concepción del tiempo como un continuo implica una codificación terminológica del mismo que no tiene nada que ver con la de un europeo ${ }^{21}$. Esto no significa que deba renunciarse a traducir alguna de las nieves esquimales al swahili, o nuestros términos "presente", "pasado" y "futuro" a algún tipo de equivalente hopi. Significa que la traducción no puede realizarse sobre la base del emparejamiento unívoco de términos, sino apelando a expedientes contextuales y culturales que permitan reflejar con cierta aproximación y un grado razonable de exactitud lo que se quiere decir en el original.

\section{"Cuando se traduce, rara vez se hace un término aislado, sino un discurso completo que plantea el reto de decidir entre varias opciones posibles"}

En esta línea, la aproximación al problema de la traducción del investigador en archivística Eric Ketelaar resulta mucho más ambigua y menos positivista, aunque, a nuestro juicio, también más acertada, teniendo en cuenta la ambigüedad misma del objeto que la investigación trata de dilucidar. De acuerdo con Ketelaar, las dificultades para emparejar adecuadamente diferentes tradiciones archivísticas no tienen su origen en la terminología, y ni siquiera en las diferencias en los procedimientos tecnológicos y administrativos. Antes al contrario, y de manera más profunda, responden a diferencias socio-culturales más amplias, que debieran dilucidarse, antes de pretender comprender una tradición archivística dada ${ }^{22}$. A partir de los criterios elaborados por el antropólogo holandés Geert Hofstede para comprender las diferencias nacionales en las relaciones dentro de las organizaciones desde el punto de vista de las aproximaciones al poder ${ }^{23}$, Ketelaar propone un aplazamiento de la asimilación, de la uniformidad y un estudio más rico y profundo de las diferencias socioculturales subyacentes a diversas tradiciones archivísticas, en una aproximación a lo que llama archivística comparada. En textos posteriores, Ketelaar ha insistido en un estudio de la diferencia, más que de la similitud, como medio para enriquecer la disciplina archivística a nivel internacional ${ }^{24}$.

Ketelaar describe adicionalmente el método de la archivística comparada como similar al método de la etnología, y sugiere la articulación de una especialidad etnológica llamada "etnología archivística"25. Esta sugerencia ha sido bien recibida por algunas comunidades archivísticas, y de unos años a esta parte se han venido realizando estudios acerca de asuntos tales como las diferencias de concepción de los sistemas de información dependiendo de las realidades nacionales, los métodos de creación de documentos en ámbitos profesionales específicos, o la concepción del archivo y del trabajo del archivero también en determinados ámbitos profesionales, utilizando como método de exploración el procedimiento etnográfico y las técnicas asociadas: cuadernos de campo, estudios de caso, investigación participativa, entrevistas de grupo o entrevistas en profundidad, tabulación de datos cuantitativos y valoración de resultados mediante software cualitativo ${ }^{26}$. Los resultados de estos análisis han resultado muy enriquecedores para el conocimiento de sus respectivos sujetos de tal modo que, y ésta es nuestra primera sugerencia metodológica, quizá antes de intentar la compilación de algo similar a un diccionario multilingüe e ideal de concordancias entre términos, debiera fomentarse la exploración de las culturas y tradiciones en las que se utilizan, a efectos de una comprensión rica de estas condiciones de uso.

Nuestra segunda sugerencia metodológica tiene un matiz algo más positivista y se refiere a la necesidad de adoptar técnicas de análisis conceptual profundo antes de proceder a un emparejamiento de los términos que hacen referencia a los conceptos. Como se ha tratado de dilucidar en lo que precede, los términos no tienen significado por sí mismos; más bien adquieren múltiples significados dependiendo de las tradiciones disciplinares, socio-culturales y nacionales en que se utilizan. Estas tradiciones han conceptualizado sus modelos teóricos y sus prácticas profesionales de diversas maneras, y sin un análisis previo de, y una comparación entre, estas conceptualizaciones, cualquier intento de concordancia terminológica debiera resultar insuficiente. Aunque la aplicación de métodos positivistas en la teoría y la práctica no se encuentra en su mejor momen- 
to, debido parcialmente a una interpretación demasiado simplista del mencionado debate entre los modelos del ciclo de vida y del continuo de los documentos, creemos que ni una aproximación positivista simple ni una aproximación postmoderna sencilla pueden resolver por sí mismas los actuales problemas asociados a la archivística. Tal y como algunos investigadores han comenzado a anotar, es de la aplicación de aproximaciones sofisticadas y más complejas de donde se pueden extraer las mejores soluciones ${ }^{27}$.

\section{Conclusiones}

Como es natural, si para traducir o de alguna otra manera comunicar los hallazgos que se están produciendo en determinadas comunidades archivísticas y de las profesiones de la información a otras comunidades con una tradición distinta hubiera que esperar a disponer de un corpus de investigación etnográfica detallada, así como de un adecuado análisis conceptual de al menos los principales conceptos sujetos a debate, nunca se traduciría nada. Estas investigaciones deben llevarse a cabo, pero, mientras tanto, somos de la opinión de que todo empeño de comunicación debiera asumir el principio de indeterminación radical de la traducción propuesto por el filósofo estadounidense Willard van Orman Quine para el ámbito de la filosofía del lenguaje y a partir del ejemplo de un lenguaje nativo del que no se conoce ningún dato. Para este lenguaje nativo, y contando sólo con la conducta observable de sus hablantes, pueden generarse un conjunto de traducciones, todas ellas incompatibles entre sí, pero todas ellas legítimas ${ }^{28}$.

Por supuesto, este principio es un préstamo tomado de otra disciplina y sólo puede utilizarse por analogía; pero a los efectos del presente texto, cumple el cometido de mostrar que no existe la traducción perfecta y que un margen de ambigüedad no sólo es inevitable sino también deseable. De igual modo, cumple el cometido de sugerir que, a la hora de comunicar diferentes tradiciones archivísticas y de las profesiones de la información, debiera tenerse en cuenta de manera prioritaria, y en términos jenkinsonianos, no el diccionario bilingüe, sino la enciclopedia de los infinitos contextos en los que los conceptos se crean y se utilizan los términos que los reflejan.

Esta aproximación debiera contribuir a tres fines fundamentales para la archivística de comienzos del siglo veintiuno: separar el trigo de la paja, aquello para lo que se debe intentar una concordancia razonablemente exacta de aquello para lo que un grado mayor de flexibilidad sería admisible; reconciliar la inevitable globalización, también en archivística, con las necesidades de las comunidades locales; y recuperar el diálogo entre las actuales tradiciones en polémica, a saber, la del modelo del ciclo de vida y la del modelo del continuo de los documentos, con vistas a extraer lo mejor de cada una de ellas.

\section{Notas}

1. Confróntese, por ejemplo, Gilliland-Swetland, Anne J. Un paradigma perdurable, nuevas oportunidades: el valor de la perspectiva archivística en el entorno digital. Consultado en: 18-09-06.

http://archivo.cartagena.es/recursos/texto1_paradigma.pdf

2. Gilliland, Anne; McKemmish, Sue. "Building an infrastructure for archival research". En: Archival science, 2004, December, v. 4, n. 3-4, pp. 149-197.

3. Acerca de esta creciente confusión: Hurley, Chris. What, if anything, is records management?. Consultado en: 18-09-06.

http://www.sims.monash.edu.au/research/rcrg/publications/ch-what.pdf

4. Muller, S.; Feith, J. A.; Fruin, R. Manual for the arrangement and description of archives. New York: H. W. Wilson Co., 1968.

5. Jenkinson, Hilary. A manual of archive administration. London: P. Lund, Humphries and Co., 1937.

6. Schellenberg, T. R. Modern archives: principles and techniques. Chicago: University of Chicago Press, 1956.

7. Acerca del desarrollo de la teoría archivística australiana: Upward, Frank. In search of the continuum: Ian Maclean's 'Australian experience' essays on recordkeeping. Consultado en: 18-09-06.

http://www.sims.monash.edu.au/research/rcrg/publications/fuptrc.html

8. El modelo del continuo de documentos se ha difundido ampliamente en los últimos años. Se consideran sus textos fundacionales:

Upward, Frank. Estructurar el continuo de los registros: primera parte: principios y propiedades postcustodiales. Consultado en: 18-09-0. http://archivo.cartagena.es/recursos/texto0_continuum1.pdf $\mathrm{y}$

Upward, Frank. Estructurar el continuo de los registros: parte dos: teoría de la estructuración y gestión de registros. Consultado en: 18-09-06. http://archivo.cartagena.es/recursos/texto0_continuum2.pdf

Estos artículos fueron publicados originalmente en 1996 y 1997. Pueden encontrarse algunas aproximaciones posteriores más estructuradas, por ejemplo: McKemmish, Sue. "Placing records continuum theory and practice". En: Archival science, 2001, December, v. 1, n. 4, pp. 333-359.

9. Acerca de la historia de la archivística reciente se ha escrito con profusión en los últimos años, como suele suceder en tiempos de crisis:

Cook, Terry. What is past is prologue: a history of archival ideas since 1898, and the future paradigm shift. Consultado en: 18-09-06.

http://www.mybestdocs.com/cookt-pastprologue-ar43fnl.htm

Thomassen, Theo. The development of archival science and its european dimension. Consultado en: 18-09-06.

http://www.archiefschool.nl/docs/thomdeve.pdf

10. Una definición de diccionario del término recordkeeping indica que significa "la creación, uso, mantenimiento y disposición sistemáticos de los documentos para satisfacer necesidades y responsabilidades administrativas, programáticas, legales y financieras". Por su parte, records management significaría "el control sistemático y administrativo de los documentos a lo largo de su ciclo de vida para asegurar eficacia y economía en su creación, uso, tratamiento, control, mantenimiento y disposición”. Por último, archives, en primera acepción, serían "materiales creados o recibidos por una persona, familia u organización, pública o privada, en la ejecución de sus asuntos, y conservados a causa de su valor permanente contenido en la información que contienen o como evidencia de las funciones y responsabilidades de su creador, especialmente aquellos materiales mantenidos utilizando los principios de procedencia, orden original, y control colectivo".

Pearce-Moses, Richard. A glossary of archival and records terminology. Consultado en: 18-09-06.

http://www.archivists.org/glossary/index.asp

Esto desde la perspectiva norteamericana. Desde Australia se renuncia a la definición de records management y se remite del término archives al de 
records, que se define de la siguiente manera: "información registrada, en cualquier forma, incluidos datos en sistemas informáticos, creados o recibidos y mantenidos por una organización o persona en la transacción de negocios o ejecución de asuntos y conservados como evidencia de tal actividad. Archivos: aquellos documentos que se evalúan como teniendo valor continuado. (Nota: el concepto unificador de documentos que es inclusivo de documentos de valor continuado, esto es, archivos)". En el contexto australiano, recordkeeping se define como "hacer y mantener evidencia completa, exacta y fiable de transacciones de negocio. Esto incluye: la creación de documentos en el curso de la actividad de negocio y los medios para asegurar la creación de documentos adecuados; el diseño, establecimiento y funcionamiento de sistemas de gestión de documentos; la gestión de los documentos utilizados en negocios (tradicionalmente considerados como el dominio de la gestión de documentos) y en cuanto archivos (tradicionalmente considerados como el dominio de la administración de archivos)". Records Continuum Research Group: Recordkeeping Metadata Project. Glossary. Consultado en: 18-09-06.

http://www.sims.monash.edu.au/research/rcrg/research/spirt/glossary.html

11. Gilliland-Swetland, ob. cit.; Ketelaar, Eric. The difference best postponed?: Cultures and comparative archival science. Consultado en: 1809-06.

http://cf.hum.uva.nl/bai/homeleketelaar/difference.doc

\section{Ketelaar, Eric, ob. cit.}

13. ISO 15489-1:2001: information and documentation: records management: part 1: general. Geneva: International Organization for Standardization, 2001.

14. DLM-Forum. Invitation to Tender $N^{\circ}$ SG/2006 - 02/PO. Consultado en: 18-09-06.

http://ec.europa.eu/transparency/archival_policy/moreq/doc/tenderspec_ en.pdf

15. Confróntense, por ejemplo, las definiciones del Proyecto InterPares 2. Document es "una unidad indivisible de información constituida por un mensaje fijado a un soporte (registrado) de manera sintáctica estable. Un documento tiene forma fija y contenido estable". Record es "un documento realizado o recibido en el curso de una actividad práctica como instrumento o producto de tal actividad, y guardado para acción o referencia". InterPares 2 project: Terminology database. Consultado en: 18-09-06.

http://www.interpares.org/ip2/ip2_terminology_db.cfm

16. Confróntese, por ejemplo:

Terrada, María Luz; López Piñero, José M. Historia del concepto de documentación. Consultado en: 18-09-06.

http://www.ucm.es/BUCM/

Galende Díaz, Juan Carlos; García Ruipérez, Mariano. El concepto de documento desde una perspectiva interdisciplinar: de la diplomática a la archivística. Consultado en: 18-09-06.

http://www.ucm.es/BUCM/

Le Goff, Jacques. El orden de la memoria: el tiempo como imaginario. Barcelona: Paidós, 1991. ISBN 84-7509-671-9.

17. Confróntese: Duranti, Luciana. Diplomática: usos nuevos para una antigua ciencia. Carmona: S\&C, 1996.
18. Merriam-Webster Online. Consultado en: 18-09-06. http://www.m-w.com/

19. Gilliland, Anne. Ensayo de discusión sobre la naturaleza y el rol de los metadatos en la creación de documentos fiables y en la conservación de documentos auténticos en sistemas electrónicos. Consultado en: 2809-06.

http://archivo.cartagena.es/recursos/texto0_metadata.pdf

20. Dryden, Jean. "A tower of babel: standardizing archival terminology". En: Archival science, 2005, March, v. 5, n. 1, pp. 1-16.

21. Confróntese Acero, J. J.; Bustos, E.; Quesada, D. Introducción a la filosofía del lenguaje. Madrid. Cátedra, 1982. ISBN 84-376-0324-2, pp. 258-262.

\section{Ketelaar, Eric, ob. cit.}

23. Hofstede, Geert. Cultures and organizations: software of the mind: intercultural cooperation and its importance for survival. London: McGrawHill, 1991. ISBN 0077074742.

24. Ketelaar, Eric. Time future contained in time past: archival science in the 21st century. Consultado en: 18-09-06.

http://cf.hum.uva.nl/bai/homeleketelaar/timefuture.doc

25. Ketelaar, Eric. Ethnologie archivistique. Consultado en: 18-09-06. http://cf.hum.uva.nl/bai/home/eketelaar/ethnologiearchivistique.pdf

\section{Confróntese}

Oliver, Gillian. "Investigating information culture: a comparative case study research design and methods". En: Archival science, 2004, December, v. 4, n. 3-4, pp. 287-314.

Gracy, Karen F. "Documenting communities of practice: making the case for archival ethnography". En: Archival science, 2004, December, v. 4, n. 3-4, pp. 335-365.

Shankar, Kalpana. "Recordkeeping in the production of scientific knowledge: an ethnographic study". En: Archival science, 2004, December, v. 4, n. 3-4, pp. 367-382.

Trace, Ciaran B. "What is recorded is never simply 'what happened': record keeping in modern organizational culture". En: Archival science, 2002, march, v. 2, n. 1-2, pp. 137-159.

27. Furner, Jonathan. "Conceptual analysis: a method for understanding information as evidence, and evidence as information". En: Archival science, 2004, December, v. 4, n. 3-4, pp. 233-265.

Gilliland y McKemmish, ob. cit.

28. Quine, W. V. O. Palabra y objeto. Barcelona: Labor, 1968.

Alejandro Delgado Gómez, Archivo Municipal de Cartagena, 3000 Informática, Parque de Artillería, Plaza del General López Pinto, s/n. 30201, Cartagena.

alejandro@ayto-cartagena.es

\section{Leer EPI es como asistir a un curso de formación continua pero con el horario acomodado a fus necesidades.}

